\title{
El aprendizaje con multimedia interactiva a través del trabajo colaborativo e individualista en rehabilitación oral
}

Enviado: 15 de septiembre de 2021 / Aceptado: 23 de septiembre de 2021 / Publicado: 31 de diciembre de 2021

MARÍA CRISTINA SIFUENTES VALENZUELA

Facultad de Odontología, UNAM, México sifuentesvalenzuela@yahoo.com

(D) $0000-0001-6603-2951$

LUIS BERNARDO MOLINA ARROYO

Facultad de Odontología, UNAM, México bermolarr@gmail.com

\section{RESUMEN}

Los estudiantes de Licenciatura de Cirujano Dentista de la Facultad de Odontología de la Universidad Nacional Autónoma de México (UNAM) deben desarrollar, habilidades cognitivas y procedimentales que les permitan resolver los problemas de salud bucodental de la población, desde su formación en las clínicas de enseñanza. En este proceso educativo, el material multimedia en los laboratorios de simulación, es un recurso que puede apoyar a los estudiantes en la comprensión y desarrollo de destrezas antes de la atención odontológica con pacientes. El objetivo de este trabajo, fue identificar la influencia del trabajo colaborativo en el aprendizaje de un tema de rehabilitación oral, que utiliza la multimedia interactiva como recurso didáctico y la estrategia SQA, con respecto a los que realizaron trabajo individualista. Se utilizó un cuestionario como instrumento para recabar la información. El tipo de muestra fue por conveniencia. Para el análisis de la información se empleo Microsoft Excel del Paquete Office 2016 y el paquete estadístico SPSS (Statistical Package for the Social Sciences, versión 2013). Se compararon los resulta-

\section{ABSTRACT}

Interactive multimedia learning through collaborative and individualistic work in oral rehabilitation

Students of the Bachelor of Dental Surgeon of the Faculty of Dentistry of the National Autonomous University of Mexico (UNAM) must develop cognitive and procedural skills that allow them to solve the oral health problems of the population, from their training in teaching clinics. In this educational process, the multimedia material in the simulation laboratories is a resource that can support students in understanding and developing skills prior to dental care with patients. The objective of this work was to identify the influence of collaborative work on learning, at the level of knowledge and understanding of an oral rehabilitation topic, which uses interactive multimedia as a didactic resource, with respect to those who carried out individualistic work. A questionnaire was used as an instrument to collect the information. For the analysis of the information, a Microsoft Excel spreadsheet of the Office 2016 Package and the SPSS statistical package (Statistical Package 
dos obtenidos antes y después de las clases en cada grupo de estudio, así como entre los dos grupos. La muestra estuvo integrada por 88 alumnos. Los resultados obtenidos en las dimensiones de estudio: Conocimiento y Comprensión, muestran diferencias estadísticamente significativas tanto en los grupos que realizaron trabajo colaborativo, y con respecto a los que realizaron trabajo individualista. Las conclusiones más relevantes se relacionan con el mayor rendimiento a nivel de comprensión por los estudiantes del grupo que efectuó trabajo colaborativo, lo que refiere su pertinencia en la formación de habilidades y competencias debido a la incidencia que tiene este nivel en la resolución de problemas bucodentales, así como por la necesidad de continuar promoviendo mas investigaciones al respecto, en aras de respaldar su conveniencia, uso y extensión a otras asignaturas.

Palabras Clave: enseñanza multimedia, odontología, aprendizaje en equipo, enseñanza clínica. for the Social Sciences, version 2013) were used. The results obtained before and after the classes in each study group, as well as between the two groups, were compared. The results obtained in the two study dimensions: Knowledge and Comprehension, show statistically significant positive differences both in the groups that carried out collaborative work, as well as with respect to the groups that carried out individualistic work. The most relevant conclusions are related to the higher performance shown by the data at the level of comprehension by the students of the group that carried out collaborative work, which refers to its relevance in the training of the students due to the impact that this level has on the resolution of oral health problems, and the need to continue promoting more research in this regard, in order to promote this methodology considering its long-term evidence.

Keywords: multimedia instruction, dentistry, team teaching, clinical teaching.

\section{INTRODUCCIÓN}

Los estudiantes de la carrera de Cirujano Dentista en la Facultad de Odontología (FO) de la UNAM deben adquirir conocimientos y desarrollar, durante su formación, habilidades y destrezas para la realización de procedimientos odontológicos de rehabilitación en las clínicas de enseñanza con pacientes que presentan problemas de salud bucodental. Con este propósito, la estructura del plan de estudios permite que el estudiante vaya adquiriendo progresivamente estas habilidades a partir de la realización de procedimientos sencillos hasta los más complejos. Asimismo, con la intención de otorgarle seguridad y evitar iatrogenias en los pacientes, antes de iniciar actividades en la clínica, la estructura del plan de estudios incluye asignaturas preclínicas con el propósito de desarrollar en los estudiantes las habilidades requeridas a través de la realización de diferentes procedimientos en los Laboratorios de Simulación (Moya et al., 2017). Este entrenamiento en situaciones controladas permite el desarrollo de destrezas clínicas transversales, conceptos teóricos y la capacidad en los estudiantes de la resolución de problemas antes de encontrarse ante una situación clínica real (Beigzadeh et al., 2016). 
La integración de las Tecnologías de la Información y Comunicación (TIC) en el proceso de enseñanza y aprendizaje ha permitido dar respuesta a las exigencias educativas actuales y a su innovación en la generación de entornos educativos pertinentes para promover el aprendizaje significativo y competencias profesionales, al favorecer el trabajo colaborativo, con el interés mostrado por los estudiantes en la organización de la tarea, y acceso directo a la información aprovechando las características y habilidades con las que cuenta la nueva generación (Ferreiro, 2006; Castellanos, y Niño, 2018). El uso de las TIC son herramientas de apoyo que potencian distintas habilidades y competencias en los estudiantes, facilitan la construcción del conocimiento y el aprendizaje significativo (Gabilondo, 2016; Pimienta, 2012; Quessada, 2015) con la creación de situaciones didácticas y metodologías activas (Frade, 2020). Las TIC al propiciar nuevas formas de aprender, permiten ampliar y enriquecer las posibilidades educativas, lo importante es el empleo pedagógico y didáctico que hacemos en su empleo. (Ferreiro, 2008).

A partir de la investigación educativa impulsada, se han podido desarrollar modelos para favorecer el aprendizaje colaborativo, en consideración a las contribuciones positivas que esta estrategia aporta al aprendizaje y desarrollo de competencias, personales y grupales. Uno de los más conocidos es el de Johnson et. al (2000). Este modelo contempla cinco elementos esenciales para un óptimo trabajo grupal: a) interdependencia positiva, elemento relacionado con la conciencia que se genera en el grupo acerca de la participación y apoyo indispensable de cada miembro para el logro de objetivos; b) interacción promocional cara a cara, aspecto importante que contribuye a potencializar los sentidos en el proceso; c) responsabilidad y valoración personal, aspecto que promueve el compromiso para un mismo fin y conciencia de la importancia del propio rol en el grupo para el logro de metas, lo que favorece la participación activa y contribuciones de cada uno de sus miembros; d) habilidades interpersonales y de manejo de grupo pequeños, constituyéndose en un elemento esencial, ya que ello permite propiciar el conflicto sociocognitivo y la retroalimentación colectiva para la solución a los problemas, generando con ello la mejora en el funcionamiento del grupo, y aportaciones positivas de otras competencias como el respeto, la disciplina, y entrega en el trabajo colectivo en beneficio del grupo y no del individualismo y e) procesamiento en grupo, este aspecto tiene lugar cuando los miembros del grupo analizan en qué medida están alcanzando sus metas y manteniendo relaciones de trabajo eficaces, evaluación que debe promoverse en el grupo para que el proceso de aprendizaje mejore en forma sostenida (Johnson, et al., 2000).

El aprendizaje colaborativo es un modelo de aprendizaje que realiza un grupo reducido de estudiantes, activos cognitivamente, que trabajan juntos para aprender algo como grupo, por lo que la participación y contribuciones se asocia a un reconocimiento grupal, y no individual 
(García, et al., 2019; Riera, 2019). La estructuración cooperativa del aprendizaje, permite que los estudiantes se ayuden mutuamente a aprender, instituyéndose como pilares básicos de un dispositivo pedagógico que permite sumar competencias individuales heterogéneas como un ingrediente positivo para el aprendizaje de todos, conjugando esfuerzo, talentos y competencias que les permitan lograr las metas establecidas por consenso (Riera, 2019; Collazos y Mendoza, 2006).

El aprendizaje que se genera con el trabajo colaborativo en el grupo, no se opone al trabajo individual, ya que el proceso y actividad que se genera intragrupal e individualmente, permite maximizar su propio aprendizaje y el de los demás, observándola como una estrategia complementaria para fortalecer el desarrollo integral del estudiante (Collazos y Mendoza, 2006; Peña y Vizcaya, 2019).

Las contribuciones que pueden alcanzarse en el aprendizaje con el trabajo colaborativo, requiere de tres interacciones colaborativas fundamentales: a) la interactividad por todos los integrantes y la forma en que esta interacción influye en el proceso cognitivo de los miembros, b) la sincronicidad de razonamiento promoviendo una comunicación recíproca, y c) la negociabilidad en donde impere el diálogo colaborativo evitando la imposición y fomentando en un ambiente de respeto una postura argumentativa (Collazos et al, 2006). De otra manera las características, heterogeneidad e intereses de los integrantes del grupo pueden constituirse en una barrera que afecte el proceso y la eficiencia del trabajo colaborativo: falta de preparación, insuficientes habilidades sociales, inconsistencias logísticas y falta de compromiso social (Hwangji, 2020).

En contraposición, el aprendizaje individualista, se distingue por el establecimiento de objetivos que se han de conseguir mediante esfuerzos personales sin interacción con otros estudiantes (Johnson, et al., 2003), promoviendo la competitividad en el grupo e individualismo, con las consecuencias negativas que esto conlleva en la formación de competencias profesionales y habilidades personales en los estudiantes.

La investigación realizada ha mostrado impacto favorable del trabajo colaborativo sobre el aprendizaje individualista y competitivo, el logro de mayores esfuerzos para alcanzar un buen desempeño, relaciones más armoniosas entre alumnos y mayor salud mental (Johnson, et al., 2003). Además, se ha confirmado la eficacia de esta metodología cuando se asegura el cumplimiento del principio de interdependencia positiva entre estudiantes (Camilli et al., 2012). Así mismo se han hecho hallazgos donde se identifica mayor rendimiento académico con el trabajo grupal (Grimm, 2004) cuando se ha comparado con el trabajo individualista, observando diferencias significativas $(\mathrm{p}<.001)$ entre estos modelos. En otro estudio se observa el mejor rendimiento académico a pesar de tener la percepción de haber aprendido menos, en comparación con la percepción de los estudiantes con trabajo individualista (Lake, D.A. 2001). 
La influencia del trabajo colaborativo en el aprendizaje ha sido estudiada y se han encontrado diferencias pretest-postest estadísticamente significativas $(\mathrm{p}<.002)$, cuando se ha implementado la colaboración en actividades didácticas (Gull, 2015). En contraposición, un estudio realizado a partir de evaluaciones a lo largo de un ciclo escolar, centrado en la retención y rendimiento académico, ha revelado resultados desfavorables para las dinámicas de aprendizaje colaborativo en relación a estas cuestiones, (Moraga y Rahn, 2007). Sin embargo, en el mismo estudio, se destaca la buena aceptación y estimación positiva que los estudiantes han referido hacia el trabajo en equipo. En cuanto a la integración de trabajo colaborativo y las TIC, las investigaciones realizadas muestran diferencias estadísticamente significativas $(p<.005)$ sobre el rendimiento de los estudiantes en los resultados de evaluaciones realizadas antes y después de las situaciones de clase (Gutiérrez, 2015).

En el área de la salud, se han obtenido resultados benéficos en el desarrollo y optimización de las habilidades de comunicación y de pensamiento crítico, trabajo en equipo, y la capacidad de resolución de problemas (Hwangji, 2020), competencias indispensables que deben formarse en los estudiantes para lograr el perfil profesional declarado en los planes y programas de estudio. En el contexto odontológico son pocos los estudios recientes realizados para identificar los efectos del aprendizaje colaborativo y empleo de las TIC en el aprendizaje. Los reportes señalan una alta aceptación de los recursos tecnológicos por parte del alumnado, y recalcan el hecho de que el trabajo colaborativo requiere de constante motivación y dedicación por parte del docente (Domínguez, et al., 2011). En cuanto al uso de material multimedia y el trabajo colaborativo como forma de aprendizaje en el contexto de la Facultad de Odontología de la UNAM, el modelo educativo y metodologías didácticas predominantes muestran limitaciones en el empleo de estas herramientas y en la colaboración como una forma de trabajo con los estudiantes (Espinosa et al., 2013).

Por lo anterior, el objetivo de este trabajo, es identificar cómo influye el trabajo colaborativo en el aprendizaje, a nivel de conocimiento y comprensión de un tema de rehabilitación oral, que utiliza la multimedia interactiva como recurso didáctico y la estrategia SQA, con respecto al aprendizaje de los que realizaron este trabajo de forma individualista. establecidos de forma clara.

\section{MATERIAL Y MÉTODO}

En la asignatura de Rehabilitación Oral I, se consolidan los conocimientos, habilidades, actitudes y valores que desarrollará el estudiante gradualmente para la rehabilitación de la salud oral de los pacientes, por lo que el uso del recurso multimedia como material de apoyo y la 
utilización de la estrategia S.Q.A. de Ogle, por su eficacia en la construcción de significados (Valdez, 2015), se consideran idóneas para lograr el aprendizaje de los principios biomecánicos de la preparación dental y el objetivo de la unidad temática.

En este año de formación, la mayor parte de los temas que forman los programas educativos están enfocados a la educación preclínica, por lo que durante el curso se asigna horas tanto a la revisión de las bases teóricas de los protocolos de aplicación, así como para el uso del instrumental, la realización de actividades en simuladores dentales y otros recursos didácticos de práctica, para ensayar y aprender los procedimientos clínicos que serán realizados en pacientes posteriormente.

\subsection{Enfoque}

Se trata de un estudio transversal analítico comparativo, con una muestra obtenida a través de una técnica de muestreo por conveniencia a partir de la selección de seis grupos distribuidos, tres para el Grupo A. Aprendizaje Individualista y tres para el Grupo B. Aprendizaje colaborativo. La muestra original quedó integrada por 94 estudiantes, de los cuáles se eliminó el $6 \%$ por problemas en el llenado del cuestionario: tachaduras, omisiones, o dobles respuestas.

\subsection{Muestra}

En este estudio participaron 88 estudiantes, distribuidos 44 de ellos en el Grupo A Aprendizaje individualista y 44 en el Grupo B. Aprendizaje colaborativo.

\subsection{Instrumento}

Se utilizó un cuestionario para recabar la información respecto al nivel de conocimiento y comprensión del tema, mismo que se aplicó antes de la intervención y dos semanas después de haber efectuado la misma. Este cuestionario quedó estructurado por 16 preguntas, ocho para identificar el nivel de conocimiento y ocho el nivel de comprensión acerca de los "Principios biomecánicos de la preparación dental”.

En el desarrollo de las preguntas, de acuerdo con Huitt, (2011), el conocimiento se concibe como el nivel cognoscitivo en el que el estudiante recuerda o reconoce información, ideas y principios en la forma aproximada en que fueron aprendidos y la comprensión como el nivel en el que traduce, comprende, o interpreta información basado en anterior aprendizaje. 
Para la validación de contenido, se envió el cuestionario a cinco profesores expertos en la asignatura, a un especialista en prótesis bucal e implantología, así como a una pedagoga. Se hizo una prueba piloto con un grupo de estudiantes del tercer año, para respaldar la confiabilidad del instrumento, en cuanto al grado en que su aplicación repetida al mismo sujeto produce resultados iguales (Hernández, et al., 2010).

\subsection{Análisis de los resultados}

Se utilizó Excel Office 2016 para el análisis de descriptivo. La comparación de medias se realizó a través del estadístico de prueba t para muestras relacionadas empleando el programa SPSS (Statistical Package for the Social Sciences, versión 2013).

\section{PROCEDIMIENTO}

El trabajo dio inicio con la elaboración del recurso multimedia interactivo para el aprendizaje de los principios biomecánicos de la preparación dental para corona total que lleva por título principal "Preparaciones Dentales". Dentro de esta se encuentra el tema "Principios Biomecánicos de las preparaciones dentales”, que se halla subdividido por un submenú en cinco subtemas: 1.1) Preservación del Tejido dental 1.2) Forma Retentiva y Resistente 1.3) Duración Estructural de la Restauración 1.4) Integridad marginal 1.5) Preservación del periodonto. La Presentación permite la interrupción de la misma, así como la libertad en la selección de la información y la secuencia de exposición. La presentación se encuentra conformada por diapositivas con hipervínculos que llevan a los temas y subtemas que se seleccionen para su presentación, la cual tiene una secuencia programada automática con texto, audio, imágenes y animaciones para cada uno de los subtemas como las que se ejemplifican en la figura 1 y 2.

Figura 1. Submenú de los principios biomecánicos de la pr eparación dent al para cor ona total. Fuente. Elabor ación pr opia

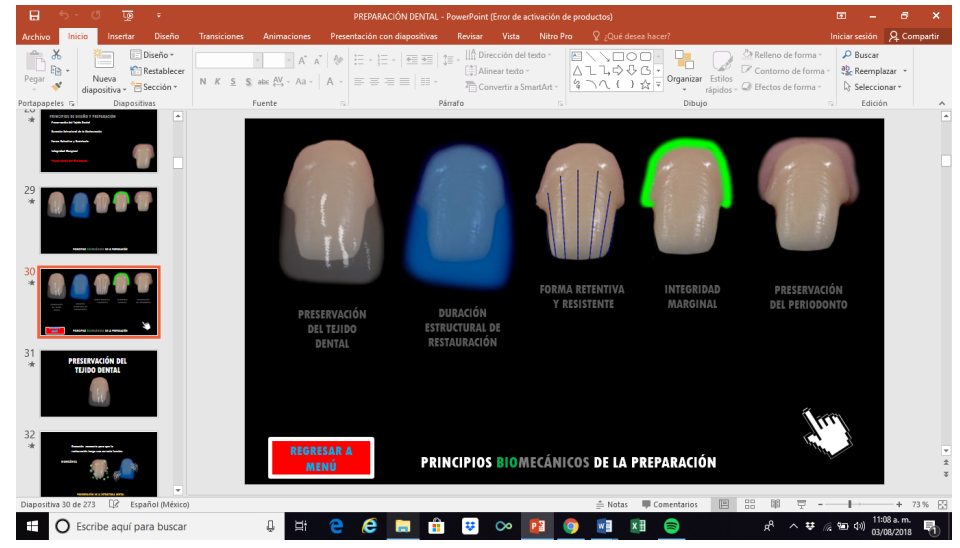


Figura 2. Imagen de una ani mación representando el principio de resistencia en la preparación dental. Fuente. Elaboración pr opia

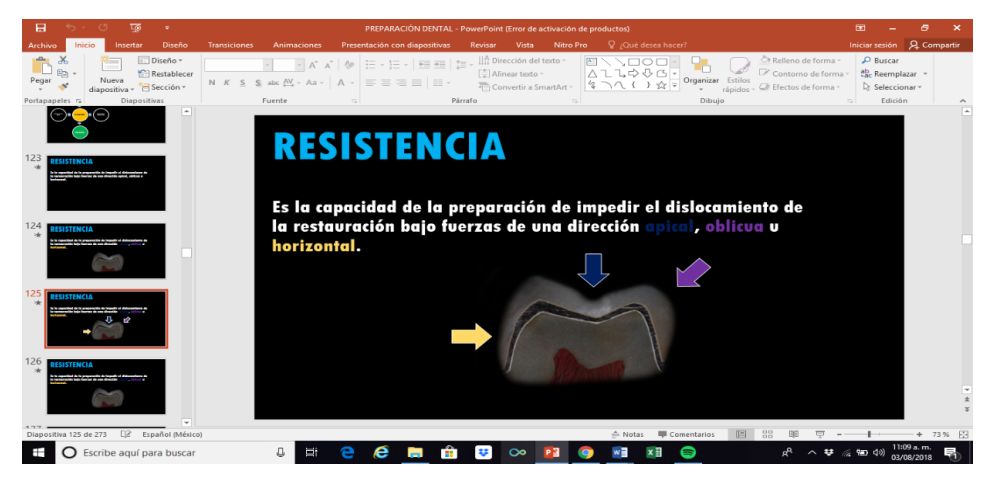

El objetivo de esta presentación es hacer una revisión acerca de los principios biomecánicos que se han de considerar al realizar preparaciones dentales para el tratamiento con prótesis fija según Shillingburg, dirigido a estudiantes de rehabilitación oral I del tercer año de la Facultad de Odontología UNAM.

De acuerdo con las situaciones de clase y el diseño del recurso multimedia, en la segunda fase se seleccionó y adecuó al recurso multimedia a la "Tabla SQA" (Sé, Quiero saber, Aprendí), estrategia de aprendizaje que permite motivar al estudio; primero indagando los conocimientos previos que posee el estudiante para después, cuestionarse acerca de lo que desea aprender, y finalmente para verificar lo que ha aprendido (Valdez, 2015).

Posteriormente se diseñó una guía de clase, donde se describen los momentos y los materiales utilizados durante las sesiones, así como las observaciones y las acciones planeadas para favorecer el trabajo colaborativo e individualista como forma de aprendizaje.

Por último, para la realización de las clases se consideró el horario y espacio de los grupos de Rehabilitación Oral I de los grupos participantes, previa autorización del profesor titular, y la fecha programada para la atención del contenido temático del programa de estudios. El contacto con los estudiantes fue presencial con una duración de dos horas por clase.

\subsection{Trabajo individual y colaborativo}

Al inicio de la clase se aplicó el cuestionario a los 88 estudiantes que participaron en la investigación para que lo respondieran de manera individual en ambos grupos (A y B).

En el grupo A Aprendizaje individualista se empleó una dinámica unidireccional. Con instrucciones para realizar la actividad de aprendizaje de forma personal. 
En el grupo B Aprendizaje colaborativo se utilizaron las instrucciones de la actividad con base en el modelo de aprendizaje colaborativo de Johnson \& Johnson (2000), previa conformación de los equipos de cuatro a cinco integrantes.

La principal diferencia entre los grupos de estudio, fue la dinámica de interacción para realizar la estrategia de aprendizaje Tabla SQA. El recurso multimedia interactivo de enseñanza, fue el mismo para ambos grupos de estudio.

Después de tres semanas de llevar a cabo las situaciones de clase, se realizó individualmente, en ambos grupos, la segunda aplicación del cuestionario.

Presentación multimedia interactiva: "Principios biomecánicos de la preparación dental"

La presentación multimedia interactiva, se diseñó con cinco secuencias de video con información acerca de cada uno de los principios biomecánicos de la preparación dental. El cuadro SQA se desarrolló para complementarse progresivamente al finalizar cada uno de los videos con duración de tres a cinco minutos, y se asignaron cinco minutos, para que los estudiantes puedan discutir y organizar la información de la forma que crean más conveniente. Esto permite que sean conscientes de la secuencia que tendrá la presentación de la información y ayuda a ir relacionando las ideas de cada uno de los principios biomecánicos revisados, presentando los que utilizan conceptos particulares al principio e ir avanzando a los que requieren de los conceptos presentados anteriormente para ser comprendidos.

\subsection{Actividad de aprendizaje Tabla SQA}

La Tabla SQA se diseñó para ser trabajada con la información del recurso, en el orden en que la información se presenta en las secuencias de video, permitiendo pausa de reflexión para el trabajo individual y cooperativo. Así como ideas detonantes plasmadas a un costado de la tabla impresa en papel. Esta actividad se lleva a cabo presentando a los alumnos un cuadro de tres columnas. La primera corresponde a lo que sé o sabemos, momento en el que se reflexiona, individual o colectivamente acerca de su experiencia relativa al tema e información al respecto. Después de plasmar por escrito lo que se sabe, se procede a llenar la columna intermedia con interrogantes acerca de lo que se quiere saber. Posteriormente, después de haber expuesto la información en cuestión se llena la última columna correspondiente a lo que se aprendió. El grupo Aprendizaje colaborativo trabajó una sola tabla entre los integrantes de cada equipo. El grupo Aprendizaje individualista trabajó una tabla por cada estudiante. 


\section{RESULTADOS}

Participaron 88 estudiantes inscritos en la asignatura de Rehabilitación Oral I. 84 \% correspondió al sexo femenino y el $16 \%$ al masculino, el promedio de edad fue de 22.3 años.

En la tabla 1, se observan las puntuaciones obtenidas antes y después de la aplicación de la estrategia didáctica, en el aprendizaje general, a nivel de conocimiento y a nivel de aprendizaje, de acuerdo con las respuestas del grupo colaborativo versus el grupo individualista.

Tabla 1. Puntuaciones obtenidas antes y después de la intervención educati va. Fuente. Elaboración propia

\begin{tabular}{lcccc}
\hline & $\begin{array}{c}\text { Antes } \\
\text { Media (DE) }\end{array}$ & $\begin{array}{c}\text { Después } \\
\text { Media } \\
\text { (DE) }\end{array}$ & tcrit & $\boldsymbol{p}$ \\
\hline Aprendizaje Gr al. colabor ativo & 40.26 & 50.43 & 4.66 & 0.000 \\
& $(9.86)$ & $(14.78)$ & & \\
\hline Aprendizaje Gr al. Individualist a & 39.82 & 42.59 & 1.31 & 0.199 \\
\hline Conocimient o colabor ativo & $(12.35)$ & $(13.09)$ & 3.06 & 0.004 \\
\hline Conocimient o individualist a & 46.51 & 54.65 & 0.34 & 0.733 \\
\hline Comprensión & $(13.17)$ & $(17.89)$ & 2.58 & 0.013 \\
\hline colabor ativo & 44.48 & 45.35 & 1.51 & 0.390 \\
\hline Comprensión individualist a & $(13.99)$ & $(12.8)$ & & \\
\hline & 34.01 & 44.48 & & \\
\hline & $(15.27)$ & $(24.44)$ & & \\
\hline & 35.17 & 39.82 & & \\
\hline & $(18.75)$ & $(19.34)$ & & \\
\hline
\end{tabular}

La DE representa la desviación estándar de las mediciones realizadas

\section{DISCUSIÓN DE LOS RESULTADOS}

Al comparar los resultados intragrupo antes y después de la intervención en los grupos Aprendizaje colaborativo y Aprendizaje individualista, respecto al Aprendizaje general las diferencias significativas, sólo se observan en el grupo Aprendizaje Colaborativo $(t=4.66 ; p=0.000)$. Resultados positivos que también se presentan en ambas dimensiones en el grupo que llevó 
a cabo el trabajo colaborativo: Conocimiento colaborativo $(\mathrm{t}=3.06 ; \mathrm{p}=0.004)$ y Comprensión colaborativo $(\mathrm{t}=2.58 ; \mathrm{p}=0.013)$.

En el análisis intergrupal Aprendizaje colaborativa vs Aprendizaje individualista, después de la implementación de la clase multimedia interactiva, se observaron diferencias estadísticamente significativas al comparar los grupos: Aprendizaje colaborativo vs Aprendizaje individualista $(t=2.246 ; p=0.030)$. Por lo que se acepta que existen diferencias significativas entre las medias de los dos grupos.

Acerca de los grupos que aumentaron su rendimiento, se encuentra coincidencia con los datos reportados de Grimm (2004) donde se establece diferencia estadísticamente significativa $(p<.001)$ entre el rendimiento de estudiantes con condiciones de clase colaborativa e individualista. De acuerdo a los datos, el aprendizaje colaborativo ha tenido un incremento mayor en las dimensiones de conocimiento y con mayor impacto en la comprensión del tema, en comparación con el obtenido con el aprendizaje individualista. En relación con los resultados intragrupales (antes y después de la clase) se encontró una diferencia significativa en el rendimiento de los estudiantes con dinámica colaborativa, resultados semejantes a los encontrados por Gull (2015), al mostrar significancia ( $\mathrm{p}=.000)$ en el desempeño de los estudiantes después de ocho semanas de actividades colaborativas en la comparación pretest-postest.

Cabe destacar que al revisar las evaluaciones individuales de cada estudiante, en el grupo Aprendizaje colaborativo, se identificaron individuos que incrementaron el nivel de aciertos hasta en un $75 \%$, datos que coinciden con lo señalado por Gabilondo (2016) y Quessada (2015), en tanto procesos que potencializan habilidades y facilitan la construcción del conocimiento y el aprendizaje significativo, así como con Frade (2020), con la creación de situaciones didácticas y metodologías activas.

Un aspecto relevante observado en el grupo durante la clase con el empleo de la multimedia interactiva fue el interés mostrado por los estudiantes sobre todo en la parte inicial de la presentación, motivación que disminuyó, conforme la sesión avanzaba, situación que refleja la respuesta que se ha identificado con el uso de los medios digitales en tanto la atracción inmediata que sienten los estudiantes por los medios digitales tecnológicos, pero se encuentran con dificultades para interactuar entre compañeros, refugiándose rápidamente en el trabajo individual (Domínguez, 2011). Esto podría verse relacionado con la falta o poco incremento de las dimensiones (Conocimiento y Comprensión) presentadas por algunos estudiantes en las evaluaciones después de la estrategia didáctica utilizada. 


\section{CONCLUSIONES}

En correspondencia con el objetivo de la investigación, los resultados obtenidos señalan un incremento estadísticamente significativo en el aprendizaje de los principios biomecánicos de la preparación dental a través del trabajo colaborativo y empleo de la multimedia para corona total. Igualmente muestra una diferencia significativa con respecto al aprendizaje bajo el modelo individualista. Tendencia que se evidencia con el mayor rendimiento que muestran los datos a nivel de comprensión por los estudiantes del grupo que llevó a cabo el trabajo cooperativo con respecto al grupo que realizó trabajo individualista. Lo que nos permite concluir que es una metodología recomendable para propiciar el aprendizaje significativo de los estudiantes y que el empleo de la multimedia, como herramienta de apoyo, es un importante recurso, si se produce asociada con los objetivos y propósito del programa educativo, para que se constituya en una estrategia didáctica.

En odontología, la colaboración para lograr un bien común es una actitud que debe promoverse en la formación de los estudiantes. Los resultados obtenidos, permiten identificar tendencias y aproximaciones en el uso de la multimedia combinada con el aprendizaje colaborativo, constituyéndose en un referente que respalda su pertinencia en el logro del perfil profesional, aunado a que puede nutrir el desarrollo de estudios futuros que respalden el empleo y extensión a otras asignaturas como aportación de este trabajo, ya que además de su incidencia en el aprendizaje y rendimiento de los estudiantes, se suma los beneficios al mejorar la capacidad de reflexión, las habilidades de pensamiento crítico y de resolución de problemas, el debate y desarrollo de competencias de comunicación, en comparación con el logrado con el trabajo individual, competencias indispensables para el desarrollo de su profesión.

Se reconoce que esta investigación tiene algunas limitaciones, como: el corto periodo en el que los estudiantes trabajaron colaborativamente, y el poco tiempo transcurrido entre la intervención educativa y la aplicación del instrumento para evaluar el aprendizaje. El cuestionario utilizado, aunque ha sido cuidadosamente elaborado para evaluar cada dimensión (conocimiento y comprensión), y construido a partir de la revisión por expertos y por la prueba piloto aplicada, se podría enfocar en una sola de las dimensiones para estudio de la influencia sobre el conocimiento o comprensión específicamente, y/o en la valoración de niveles de conocimiento superiores en correspondencia con los objetivos curriculares del programa educativo, de este u otras asignaturas.

Este trabajo de investigación tiene relevancia significativa en el contexto local, por la poca implementación del trabajo colaborativo identificado en la formación preclínica, así como la 
escasez de recursos didácticos digitales para la educación odontológica, ya que, a pesar de existir abundante material multimedia a disposición de profesores y alumnos, pocos de estos recursos tienen una orientación educativa apta para la formación preclínica.

En este contexto la realización de acciones orientadas a la capacitación digital y diseño de materiales se señalan como referentes importantes para aprovechar estas herramientas, así como para el desarrolla de recursos multimedia alineado a los programas de estudio, y de esta manera contribuir a una innovación eficiente en la práctica docente.

\section{REFERENCIAS BIBLIOGRÁFICAS}

Beigzadeh, A., Bahmanbijri, B., Sharifpoor, E., \& Rahimi, M. (2016). Standardized patients versus simulated patients in medical education: are they the same or different. Fournal of Emergency Practice and Trauma, 2(1), 25-28. https://doi.org/10.15171/jept.2015.

Camilli, C., López, E., \& Barceló, M. (2012). Eficacia del aprendizaje cooperativo en comparación con situaciones competitivas o individuales. Su aplicación en la tecnología: Una revisión sistemática; Enseñanza \& Teaching-30, 81-103.

Castellanos-Ramírez, JC., \& Niño, SA. (2018). Aprendizaje colaborativo y fases de construcción compartida del conocimiento en entornos tecnológicos de comunicación asíncrona. Innovación educativa (México, DF), 18(76), 69-88.

Collazos, César Alberto, \& Mendoza, Jair (2006). Cómo aprovechar el "aprendizaje colaborativo" en el aula. Educación y Educadores, 9(2),61-76.

Domínguez, A., Casado I. \& Romero M. (2011) Aprendizaje colaborativo en primer curso de odontología. Uso de la Wiki en Salud Pública. VII Jornadas de Campus Virtual UCM. 2011, p.123-128.

Espinosa, O., Martínez, A., Díaz, F. (2013). Formas de enseñanza y evaluación utilizadas por los docentes de Odontología: resultados y su clasificación psicopedagógica. Investigación en Educación Médica; 2,183-192.

Ferreiro, R. (2006). El reto de la educación del siglo XXI: la generación N. Apertura, 12, 72-85.

Ferreiro R, Vizoso E., (2008). Una Condición Necesaria en el Empleo de las TICs en el Salón de Clases: La Mediación Pedagógica. Posgrado y Sociedad. 2008; Vol. 8 No. 2 .72-88.

Frade, L. (2020). Diseño de situaciones didácticas. Estrategias de enseñanza-aprendizaje desde el contexto. Mediación de Calidad. 512 
Gabilondo, C. (2016). Video científico como soporte al proceso enseñanza-aprendizaje. Universidad Politécnica Salesiana, II, 254-264.

García R., Traver J. \& Candela I. (2019). Aprendizaje cooperativo. Colección acción social. Cuaderno 11. Edit. CCS. Pp. 79.

Grimm, D. (2004) Individual learning versus group learning in a suburban second-grade classroom. Theses and Dissertations. 1156, https://rdw.rowan.edu/etd/1156

Gull F., \& Shehzad S. (2015) Effecto of Cooperative Learning on Students Academic Achievement. fournal of Education and Learning. Vol. 9 (3) pp. 246-255.

Gutiérrez, C. (2015) Análisis comparativo de metodologías de aprendizaje colaborativo Jigsaw y Aprendizaje basado en problemas, haciendo uso de objetos de Aprendizaje reutilizables para el aprendizaje de la geometría, en alumnos de primero medio. Universidad de Chile, 17-18.

Hernández, R., Fernández, C. \& Baptista L. (2010). Metodología de la investigación. 50 Ed. McGraw-Hill. México.

Huitt, W. (2011). Bloom et al's taxonomy of the cognitive domain. Educational Psychology Interactive. Valdosta, GA: Valdosta State University. Disponible en: http://www.edpsycinteractive.org/topics/cogsys/bloom.html

Hwangji, S. (2020). Collaborative learning needed for healthcare management education. International Journal of Economics, Business and Management Research. Vol. 4, No. 09.

Johnson, D. W., Johnson, R. T., \& Stanne, M. B. (2000). Cooperative learning methods: A meta-analysis. Minneapolis: University of Minnesota.

Johnson, D.W., Johnson, R.T., \& Holubec, E.J. (2003). Aprender cooperando: el aprendizaje cooperativo en el aula. Recuperado de https://www.guao.org/sites/default/files/biblioteca/El aprendizaje cooperativo en el aula.pdf \%0Ahttp://books.google.es/books?id=XjhfPAAACAAJ.

Lake, D. A. (2001). Student performance and perceptions of a lecture-based cours compared with the same course utilizing group discussion. Physical Therapy, 81(3), 896-902.

Moraga, R., \& Rahn, R. (2007). Studying knowledge retention through cooperative learning in an operations research course. IIE Annual Conference and Expo 2007-Industrial Engineering's Critical Role in a Flat World - Conference Proceedings, 1161-1166.

Moya, P., Ruz, M., Parraguez, E., Carreño, V., Rodríguez, A.M., \& Froes, P. (2017). Efectividad de la simulación en la educación médica desde la perspectiva de la seguridad de los pacientes. Revista Medica de Chile, 145(5), 14-26. 
Peña, H. \& Vizcaya, T. (2019). Efectos del aprendizaje cooperativo según el modelo de Johnson \& Johnson-Holubec sobre el rendimiento estudiantil en el contenido de estequiometría. Rev Observador del conocimiento. Vol. 4, No. 2. Mayo agosto 2019. 8-100.

Pimienta, J. (2012)Estrategias de enseñanza aprendizaje docencia universitaria basada en competencias $1^{a} E d$. México: Pearson.

Quessada, M. J. (2015). Creación de videos educativos como estrategia didáctica. Actualidades Investigativas en Educación, 15(1), 1-19.

Riera, G. (2017). El aprendizaje cooperativo como metodología clave para dar respuesta a la diversidad del alumnado desde un enfoque inclusivo. Revista Latinoamericana de Educación Inclusiva. 134149.

Revelo-Sánchez, O., Collazos-Ordoñez, C.A., \& Jiménez-Toledo, J.A. (2018). El trabajo colaborativo como estrategia didáctica para la enseñanza/aprendizaje de la programación: una revisión sistemática de literatura. TecnoLógicas, vol. 21, no. 41, pp. 115-134.

Solanki, K. \& Acharya, P. (2019). Effectiveness of collaborative learning on reading comprehension. International Research fournal of Management Sociology \& Humanity. Vol. 10 Issue 5. 34- 240.

Valdez, C., (2015). Compendio de estrategias de aprendizaje. 1ª Edición. Universidad Mariano Gálvez, Guatemala. 3 